\title{
Megarian Variable Actualism
}

\section{Toby Friend ${ }^{1}$}

Received: 30 November 2020 / Accepted: 10 June 2021 / Published online: 29 June 2021

(c) The Author(s) 2021

\begin{abstract}
Megarian Actualism is the denial of unmanifesting powers. Aristotle called such a view 'buffoonery' and dispositionalists have provided compelling reasons for the contrary platitude that powers need not manifest. Even so, drawing on extant treatments of quantitative powers I'll suggest that many of the powers which feature in quantitative lawlike equations are plausibly interpreted as Megarian. This is because the powers described by such equations are best understood as being directed towards all the values of exhaustive manifestation variables. I'll discuss the prospects for generalising these Megarian characteristics to powers not typically represented in strict quantitative terms. The result will be a strong basis for a scientifically informed and plausible dispositionalist account: Megarian Variable Actualism.
\end{abstract}

Keywords Dispositions · Powers · Megarian Actualism · Quantitative powers · Multi-track powers

\section{Introduction}

Dispositionalism is the view that there exist 'powers', properties which essentially confer dispositions on their bearers. Powers confer dispositions by virtue of being 'directed' (Martin 2008; Molnar 2003; Tugby 2013), 'for' (Martin and Pfeifer 1986), 'pointing beyond' (Heil 2003), 'oriented towards' (Tugby 2013), 'pointing' to (Handfield 2010) or simply being 'related with' (Place 1999) some outcome.

These further outcomes are referred to as a power's 'manifestation' (though they need not be observable), an expression or 'essential display' of the power (Ellis 2000). Powers, then, confer dispositions essentially because they could not fail to be directed to their respective target manifestations. Manifestations are naturally thought of as an

This article belongs to the topical collection "New Foundations of Dispositionalism", edited by Andrea Raimondi and Lorenzo Azzano.

Toby Friend

toby.friend@bristol.ac.uk

1 Department of Philosophy, University Bristol, Cotham House, Bristol BS6 6JL, UK 
'effect' of powers (McKitrick 2010) which may itself be an outward behaviour or a contribution to outward behaviour alongside other effects.

What more can be said about powers' directedness? One thing almost universally accepted is that anything with a power directed towards a manifestation $M$ can instantiate $M$ (i.e. can manifest the power). ${ }^{1}$ Fragile objects can break, sonorous materials can make a sound, malleable things can change shape, etc. A power's directedness therefore implies a possibility for manifestion. Something else which almost no dispositionalist account at least since Aristotle has disputed is that powers need not manifest. (I permit myself the shorthand that it is the power that may or may not manifest, but more precisely speaking it is things which instantiate the power which manifest that power). Fragile objects needn't always break, sonorous materials needn't always be making a sound, malleable things needn't always change shape, and so on. Indeed, most dispositionalists think objects with such powers need not ever manifest. A power's directedness then implies a possibility for, but not the necessity of, manifestation.

The possibility for a power to go 'unmanifested' is an oft-cited feature of dispositionalism. Martin remarks, for instance, that 'we must see that dispositions are actual, though their manifestations may not be' (1994, p. 1). Heil says that 'a disposition [power] can persist unmanifested and, in the right circumstances, be unmanifestable' (2005, p. 344). Molnar assumes the independence claim that 'powers can exist in the absence or in the presence of their manifestations and so are ontologically independent of the occurrence of the manifestations' (Molnar 2003). Tugby (2013) confirms that dispositionalists have reached something of a consensus in this regard, referring to the view that particulars can have powers even if they never manifest it a 'central dispositionalist platitude'.

As with many points of consensus among dispositionalists, their routes go back to Aristotle. Aristotle talked of monadic properties which are 'pros ti' (towards) something else (Aristotle 1994, 6a36-b3), where such properties had potentialities to act or bring about change. In Book $\Theta$ of his Metaphysics he mocked the suggestion that powers always manifest.

Now there is a chestnut that enjoys widespread popularity, not least in Megara, to the effect that $x$ is capable of building/doing the $F$ only when it actually is/does the $F$. So the non-builder is no bearer of a potentiality for building - the only such bearer is the builder when engaged in his building, etc., etc.

Exposing the idiocy of this buffoonery will not long detain us. (1998, Book $\Theta$, part 3)

The recipient of Aristotle's insults has come to be known as 'Megarian Actualism'; evidently it is the exact antithesis of Tugby's central dispositionalist platitude:

Megarian Acutalism. Particulars must always manifest their powers.

As roundly rejected as it is, and at the risk of suffering the insults of The Philosopher himself, I'm here going to make a case for Megarian Actualism; that is, at least for a certain class of powers. I'll begin first by detailing what I take to be the main concerns

\footnotetext{
1 I consider to be an outlier the view suggested by Jenkins and Nolan (2012) according to which there may be powers with impossible manifestations.
} 
with Megarian Actualism, drawing on the few explicit engagements with it (Sect. 2). Then, drawing on extant treatments of quantitative powers I'll suggest that many of the powers which feature in quantitative lawlike equations are plausibly interpreted as Megarian. This is because the powers characterised by such equations are best understood as being 'quantitatively multi-track', in that they are directed towards all the values of exhaustive manifestation variables (Sect. 3). I'll then show why these quantitative powers do not suffer the criticisms raised in the previous section and give further support to the plausibility of permitting every value of the manifestation variable to count as genuine manifestation by considering the extreme case of zerovalue manifestation explicitly (Sect. 4). I'll then move on to discuss the prospects and motivation for a thesis of Megarian Variable Actualism, which generalises these Megarian characteristics to powers not typically represented in quantitative terms (Sect. 5). The conclusion restates the case for a scientifically informed and plausible Megarian account of dispositionalism (Sect. 6).

Before continuing, some necessary path-clearing is in order. Dispositionalists disagree on a lot about which the discussion that follows will be entirely silent. Though there are points of contact, my case for Megarian Actualism is largely independent of whether powers are tropes or universals, whether all or only some properties are powers, and whether powers are 'pure' or have also some (underlying or dual-aspect) qualitative nature. For brevity I will also make some terminological decisions which shouldn't affect the overall arguments. I will, for instance, continue to talk of further properties relevant to how a power manifests as being 'stimuli', even if such properties would be better conceived as manifestation partners. I'll not pass judgement on whether all or only some powers have stimuli. I'll also continue to use the word 'power' to stand for the property instantiated which confers dispositions on their bearers. However, this contrasts with some of the below and above quotations in which 'disposition' is used as a synonym for 'power'.

\section{The trouble with Megarian Actualism}

It is so widely assumed that powers need not manifest that explicit engagement with Megarian Actualism has been scarce. There have, however, been a few critical remarks. I'll here mention three, respectively from Bird, Molnar and Aristotle, which I take to cover the broad range of philosophical issues one might take with the position. I'll then end with a more common-sense concern.

Bird discusses Megarian Actualism in the context of his discussion of unrealised possibilities, e.g. those expressed by non-trivial counterfactuals. His reasoning runs as follows. We should accept the possibility of things which don't in fact occur, and so the truth of non-trivial counterfactuals. Modal Realists make sense of this by endorsing the reality of non-actual possible worlds; the alternative is to ground unrealised possibility in actuality. One promising way to do this, Bird suggests, is to have powers ('dispostions') be parts of the actual world. He remarks, however, that

the Megarian [A]ctualist denies that a disposition can exist without being manifested, i.e. they deny, 
(B2) unmanifested dispositions can be parts of the actual world.

[And so, ...] if the [Megarian] actualist gives counterfactuals a standard possible-world semantics, then counterfactual propositions collapse into the corresponding material conditional. [...] This requires a denial [also] of,

(B1) non-trivial counterfactuals can be made true by facts in the actual world alone. (Bird 2007, p. 109)

One might query the implicit premise that the Megarian Actualist must accept that nontrivial counterfactuals must be grounded in powers, if at all. However, in the context of dispositionalism that can seem like a reasonable assumption. In sum, the objection to Megarian Actualism which can be extracted from Bird's discussion amounts to the thought that the view trivialises modal talk, restricting the range of possibility down to jut what is actual. Assuming, with Bird, that non-trivial possibilities are worth making sense of, this observation seems extremely problematic for the Megarian.

Molnar's criticism of the view is concerned more with explanation than modality. Particulars, he notes, aren't attributed a certain power merely in virtue of manifestating, since attributing the power is supposed to help explain manifestation. Yet according to Molnar,

it is hard to understand the concept of a power that exists precisely when it manifests and for exactly as long as it manifests [...] Megaric powers are quite incapable of playing any part in explaining the causal interactions in which they participate, since they are themselves ontologically parasitic on those interactions. (2003, pp. 97-98)

The explanatory demand on powers which Molnar notes seems entirely correct. It is evident, for instance, when we explain why some occurrences are liable for some objects and not others. Anvils aren't liable to break whereas vases are because vases but not anvils are fragile. The concern with Megarian Actualism, however, is that since powers are understood to only be present when their manifestations are, they are ontologically dependent ('parasitic') on them. This seems to remove any possibility for the power to explain, when instantiated, the manifestation. Indeed, the explanation would rather appear to have to go the other way around, with manifestations explaining the presence of the power.

The third objection for us to consider is Aristotle's own. It is closely related to Molnar's but worth setting out independently.

An immediate, and undeniable, upshot [of Megarian Actualism] is that, if a man is not engaged in building, he is not even a builder! (Assuming, as I take it that we may, that to be a builder is to have a power for building.) [...] But we assume that it is impossible to have such skills without having learned and acquired them at some time and that, thereafter, it is impossible not to have them unless one has at some stage dis-acquired them (and the options here are forgetting, physical or other impariment or mere lapse of time [...]) So, [according to Megarian Actualism] the moment a man lays down his trowel, he will no longer have the skill. But, of course, he may very well start building again at any moment. But 
when (and how) will he have acquired the skill to do so?! (1998, Book $\Theta$, part 3)

The worry Aristotle is voicing seems to be one about the general non-triviality of acquiring and loosing a power. Prima facie, a builder can acquire the power to build a house by growing strong and becoming educated in the right way. Moreover, it seems he or she can loose that power by becoming physically or mentally impaired. However, since the Megarian has it that powers are only instantiated when they're manifesting, it appears they must deny these non-trivial conditions of acquisition and loss. A builder, for example, does not re-gain strength and become re-educated every time they pick up a trowel. Nor do they become impaired every time they put it down. Not only does it seem, then, that the putative criteria of acquisition and loss of the power cannot be the real ones for the Megarian, but they will struggle to say what the acquisition and loss conditions must be. This is why it can seem like the manifestation itself must play a role in explaining acquisition and loss of the power, lending more weight to Molnar's criticism that the Megarian must get the order of explanation wrong.

Beyond these three 'philosophical' objections I take it that there may just be a lingering sense that the Megarian has failed to take account of the data. Fragile vases are not always breaking, malleable clay is not always changing shape, sonorous metals are not always making a sound, etc. More than this, we seem to be able to say when these objects will not do these things; namely, when they are not being appropriately stimulated, e.g. subject to pressure or stress, or when that stimulation is being appropriately masked (Johnston 1992), e.g. protected in styrofoam. The Megarian seems to have to deny all these kinds of claim. So even if the concerns over modality, explanation, acquisition and loss can be overcome, the Megarian will still have to justify why these aren't sensible things to say, or else show why they don't have to deny them. In Sect. 5 I'll explain a way for the Megarian to take the second of these options with respect to powers in general. But first, I want to turn to a different class of powers and show both (Sect. 3) why they are straightforwardly conceived as Megarian and then (Sect. 4) why none of the foregoing objections work against them.

\section{Megarian powers in quantitative equations}

Many quantitative lawlike generalisations used across the sciences seem to involve powers. Here's a small sample (in no particular order). The specific heat formula is

$$
Q=c m \Delta T
$$

and concerns the disposition conferred on an object by its specific heat capacity $c$, along with mass $m$ and some amount of heat $Q$ to manifest a change in temperature $T$. Ohm's law can be expressed

$$
J=\sigma E
$$


and concerns the disposition conferred on an object by its conductivity $\sigma$ to manifest a current density $J$ in response to an electric field $E$. The total force law is

$$
F_{n e t}=m a
$$

and concerns the disposition conferred on an object by its inertial mass $m$ (a form of resistivity to motion) to manifest acceleration $a$ under an applied net force $F_{\text {net }}$. The logistical growth model is

$$
\frac{d P}{d t}=r P\left(1-\frac{P}{K}\right)
$$

and concerns the disposition conferred on an environment by its carrying capacity $K$, along with a population $P$ 's growth rate $r$ to manifest a certain change in population over time $\frac{d P}{d t}$. Hooke's law is

$$
F_{\text {load }}=k \delta
$$

and concerns the disposition conferred on an object by its stiffness $k$ to manifest some extension $\delta$ in response to the load force $F_{l o a d}$. The price-demand curve is

$$
\alpha=\frac{\partial Q / Q}{\partial P / P}
$$

and concerns the price elasticity $\alpha$ of a commodity to manifest a change $d Q / Q$ in demand in response to changes $d P / P$ in price. Finally, the Coulomb force on a particle is given by

$$
F_{\text {elec }}=q E
$$

and concerns the disposition conferred on an object by its charge $q$, along with the electrical field $E$ at its location to manifest a certain exerted electrostatic force $F_{\text {elec }}$.

Though one or other of the referenced powers might be brought into question by metaphysical considerations, I will take the policy of assuming they are all to be incorporated within the dispositionalist framework. I will not be considering, therefore, the possibility that powers are only found at the 'fundamental' level (pace Bird 2007). Rejecting the legitimacy of the examples would, I think, do a disservice to the inferential support dispositionalism is fit to gain from scientific explanatory practices. For the scientific validity of equations like the foregoing appear to be among the best evidence of dispositionalism. Moreover, a central theme of dispositionalist advocacy is the ability of dispositional properties to illuminate known characteristics of lawlike generalisations such as those listed above (Harré and Madden 1974; Cartwright 1983, 
1999, 2019; Chakravartty 2003; Hüttemann 2004, 2014; Kistler 2012, 2020; Vetter $2015 .^{2}$

So let's grant the legitimacy of the above powers. ${ }^{3}$ I take it that each specific equation offered above is characteristic of the respective power. What this means is that the essential directedness encoded by any instance of the power variable is captured by its characteristic equation(s). This has a number of crucial consequences. First, characteristic equations will typically include a variable for the characterised power (henceforth 'power variable') itself and necessarily a corresponding variable for the manifestation (henceforth 'manifestation variable'). For example, the specific heat formula tells us that determinate specific heat capacities manifest temperature changes $\Delta T$; Ohm's law tells us that the determinate conductivities manifest currents $I$. Variables which are neither power variable nor manifestation variable will then be further relevant factors (influences of the manifestation variable) to which we might attribute the status of 'stimulus variables'. Second, even if the characteristic equations are not accurate in all circumstances (see the below discussion on idealisation), they must capture a relationship of directedness which applies in any circumstance in which the power they characterise is present. ${ }^{4}$ So, for example, although the logistic equation may not be accurate in many circumstances, the directedness between carrying capacity (the characterised power variable) and population change (the manifestation variable) always holds whenever the power is instantiated. In the above 'scientific cases' at least, a power's directedness therefore amounts to a relationship described by a possibly idealising characteristic equation (though a power variable may of course feature in many other scientifically useful equations). ${ }^{5}$

Now, we know that both power variables and manifestation variables can be directly attributed to objects: resistors exhibit conductivities and current densities, environments exhibit carrying capacities and population changes, etc. I also take it that it is natural to think of the associated power of each object being that single determinate value of the power variable which in fact gets instantiated at any moment. A particular resistor, say, may be said to have the determinate quantitative conductivity of $10 \mathrm{Sm}^{-1}$, an environment may have the determinate quantitative carrying capacity of 500 for some organism, a steel spring may have the determinate constant of $600 \mathrm{kgs}^{-2}$, etc. In any given circumstance, we may therefore expect the manifestation variable to take a specific value too. However, it is certainly implausible that the characteristic manifes-

\footnotetext{
${ }^{2}$ Keeping reference to 'lawlike generalisations' allows us to include Mumford (2004) in the list as well, since presumably Mumford will agree there are such generalisations even if no laws themselves.

${ }^{3}$ My case for Megarian Variable Actualism relies on these being confirming examples, but the consistency of the account and many of its benefits would not be lost if we were to have to reject them.

${ }^{4}$ This point is therefore consistent with but comes short of entailing the dispositionalists' widespread endorsement of the metaphysical necessity of laws (Shoemaker 1979; Swoyer 1982; Ellis and Lierse 1994; Bird 2007). By labelling the equations as 'characteristic', we imply that the characterised relations of directedness hold of metaphysical necessity, not the numerical solutions themselves (though they might). If the dispositionalist wants to endorse powers which are essentially described by idealisation and/or ceteris paribus laws, this seems like an unavoidable clarification.

5 A power variable will have multiple characteristic equations if it is genuinely qualitatively multi-track (see the comparison with quantitatively multi-track powers later on), as charge arguably is (Kistler 2020). A power variable may also appear as a stimulus variable in an equation which characterises some other power, e.g. the growth rate in the logistic equation.
} 
tation any determinate quantitative power is directed towards is a single value. After all, to pick any specific value of the manifestation variable would be entirely arbitrary. To take a single example, electric charge $e$ (approx. $1.6 \times 10^{-19} \mathrm{C}$ ) is no more directed towards any specific value of electrostatic force than any other value; it may in fact be manifesting $5 p N$ in some co-ordinate system, but it could have manifested a different force.

Given that we must avoid unreasonably biasing some values of manifestation over others there appear to be two options. The problematic option is to posit an infinity of 'single-track' powers associated with the predication of a determinate value of a power variable, such as 'has electric charge', each with different stimuli and being directed towards distinct values of the manifestation variable (this seems to be the view of Bird 2007). Both Kistler $(2012,2020)$ and Vetter $(2012,2015)$ have persuasively argued against this idea. The problem is that by endorsing a plethora of single-track powers for some attribute, like having electric charge, one fails to account for the evident unity and modal profile implied by the attribution of that attribute. It is only, they argue, if one takes the second option of positing a single 'multi-track' power which on its own is directed to all the possible values of the manifestation variable that can one make sense of these characteristics. Hence Vetter remarks that a single multi-track power of electric charge

fixes facts about the more specific dispositions which are not fixed by those [single-track] dispositions themselves, even taken in conjunction. These are facts about the co-instantiation of the more specific dispositions: the fact, for instance, that if anything has one of the specific dispositions corresponding to electric charge, it has all the others too. (Vetter 2015, pp. 57-58)

And in general, Kistler observes that

the hypothesis that there is a real powerful property underlying a set of dispositions $D_{i}$, has the form of an inference to the best explanation. If there is a unique powerful property that is the causal basis of a set of dispositions $D_{i}$, it provides a unique and unifying explanation of their manifestations $M_{i}$. (Kistler 2012, p. 125)

Crucially, the case made in these quotes by Vetter and Kistler is for powers which are multi-track by being directed towards the range of values of a quantity. Traditionally, the dispute between whether or not powers are single or multi-track has revolved around whether powers manifest in qualitatively different ways (e.g. Williams 2011). And Kistler and Vetter have both suggested that parallel arguments carry over to to the qualitative case. But the argument just considered encourages at the very least the view that some powers unify a full range of quantitative values of an exhaustive but otherwise qualitatively homogenous manifestation variable under the target of a single power. Putting the debate over qualitative multi-track powers aside, I'll refer to these powers of more recent interest as 'quantitatively multi-track' (henceforth 'QMT'). ${ }^{6}$

\footnotetext{
${ }^{6}$ For Kistler, it will turn out that some powers are both (e.g. charge). However, QMTs are arguably more plausible than qualitatively multi-track powers. For an argument against the latter, see Lowe (2010).
} 
Kistler and Vetter's interpretations of QMT powers differ in certain respects. For Kistler, multi-track powers are described in terms of a plurality of counterfactuals whereas in Vetter's terms, quantitative powers are described in terms of a unconditional possibility—or 'potentiality' — for a plurality of material conditionals. ${ }^{7}$ But regardless of the difference, what is crucial is that under either interpretation, every possible value of a QMT power's manifestation variable covered by the power's characterising equation is an equally legitimate instance of the manifestation of the power. ${ }^{8}$ Kistler gives the example of the conductivity of a piece of copper which

can manifest itself in infinitely many ways: by giving rise to electric current of density $J_{1}$ in a situation with electric field $E_{1}$, to $J_{2}$ in the context of electric field $\mathrm{E}_{2}$, etc. All these dispositions to manifest by current densities $\mathrm{J}_{i}$, given field $\mathrm{E}_{i}$, can be expressed by a single law stating that $\mathrm{J}$ is proportional to $\mathrm{E}, \mathrm{J}=\sigma \mathrm{E}$. (2012, p. 122)

Because the variable $J$ is exhaustive, the copper's conductivity is manifesting for any value of current density it can instantiate. Vetter's example of the electrical charge of an electron is similar. Because the variable for electrostatic force is exhaustive, an electron's charge is manifesting for any value of force it experiences.

Though they fail to draw the conclusion, this feature of both Kistler and Vetter's interpretations is strongly suggestive of Megarian Actualism. Since all possible values of the manifestation variable count as genuine manifestations, and since the determinate instances of these power variables cannot fail to be exhibiting some or other value of their manifestation variables, they will therefore always be manifesting. Notice that this Megarian outcome holds even if, as both Vetter and Kistler suspect, the respective powers' characterising equations are not universally true. Understandably, Kistler and Vetter are keen to make space, respectively, for ceteris paribus and idealisation conditions, i.e. conditions which undermine the precision and/or strictness of the function defining which manifestation values correspond to which powers and stimulus values. Vetter explicitly recommends (pp. 288-290) that the operator for idealisation laws be treated as 'less than maximal', where maximality corresponds with metaphysical necessity and anything weaker permits actual cases which do not obey the material conditionals. And Kistler suggests that, due to the possibility of masking, a power's implication of a value for manifestation under some determinate stimulus cannot be expected to be hold in all cases. As a consequence, neither interpretation preserves the crucial-and surely correct-point that even in non-ideal circumstances, any determinate instance of the power variable will exhibit some value of the manifestation variable. A piece of copper wire interfered with by a heavy magnetic field may not exhibit a current density in accordance with $\mathrm{J}=\sigma \mathrm{E}$, but it will exhibit some value for current density. Similarly, an electric charge $e$ interfered with by relativistic effects may not experience an electrostatic force in accordance with $F_{\text {elec }}=q E$, but it will

\footnotetext{
7 One wonders whether Vetter can really maintain the materiality of the embedded conditionals. For classic reasons, the description would fail to serve as an analysis at least. However, if the conditionals are counterfactuals and the potentiality operator $(\Delta)$ is interpreted as that of strict necessity then the two descriptions will be equivalent (assuming, as is surely the case, that Kistler's descriptions are supposed to be necessary).

8 Worried about the zero-values? These will be discussed at length in Sect. 4.
} 
experience some value of force. ${ }^{9}$ These facts are a simple result of the fact that the manifestation variable in each case must be instantiated whenever the power is present (since the relevant equations are characteristic) and is exhaustive of the possible states an instance of the respective power variable can be in.

We therefore have the makings of a case for Megarian powers in the context of the kinds of lawlike characteristic equations given above. The case builds on the observation that certain equations characterise the directedness of power variables towards manifestation variables. It was observed that determinate instances of those variables cannot be directed at any specific value over any others, which suggests that determinate instances are either accompanied by a multiplicity of single-track powers each directed at one possible value of the manifestation variable, or else have a single unifying QMT power equally directed at every possible value of the manifestation variable. Kistler and Vetter have given us good reason to prefer the latter option. But something they failed to point out is that, in the cases considered, since their characteristic manifestation variables must be instantiated whenever the power variable is, and since it must take some value, the powers are always manifesting.

Admittedly, this path to Megarian Actualism may not be the way typically imagined. The criticisms noted in Sect. 2 reveal that powers' manifestations have typically been conceived of in binary terms: either a power manifests a single determinate property $M$, or it doesn't. Given such a conception there can be no difference between a power failing to manifest $M$ and it failing to manifest simpliciter. However, once we acknowledge QMT powers the possibility of a difference opens up. The determinate instance of some power variable can fail to manifest any particular value of its manifestation variable without it failing to manifest by taking some other value. And indeed, since the above QMT powers always manifest some value of the manifestation variable that's sufficient to satisfy the definitions of Megarian Actualism found in Aristotle (1998, see above), Molnar (2003, pp. 94-95) and Bird (2007, p. 109).

One might complain, however, that calling these QMT powers 'Megarian' nevertheless misses the point of what its critics were trying to get at. If that's right, then the powers just considered may be Megarian in letter though not in spirit. Yet one might reasonably wonder what Megarian Actualism would have to amount to in the context of quantitative powers if not the QMT view just sketched. Certainly, one should not expect the Megarian view to be that a determinate instance of a power variable is manifesting all possible values of the manifestation variable all the time-Megarianism is supposed to sound strange, perhaps, but not incoherent!

I will continue to treat the above powers as deserving the title 'Megarian' if, that is, it is indeed true that every value of the manfiestation variable counts as a genuine manifestation, since given that assumption the manifestation variable is always instantiated. However, a further reason to doubt the correctness of calling these QMT powers 'Megarian' rests on the failure of just this assumption. For I take it that one might reasonably query treating the specific case in which the manifestation variable takes a zero-value as one of genuine manifestation. This concern effectively amounts to the last objection noted in Sect. 2 that the Megarian isn't able to make sense of

\footnotetext{
9 It is arguable whether there is such a thing as electrostatic force within relativistic physics. Nevertheless, whatever force variable replaces $F_{\text {elec }}$ will be subject to the same issue.
} 
the common-sense data about non-manifestation. I'll have something to say by way of response to this in the next section after having first considered the former three 'philosophical' criticisms of Megarian Actualism.

\section{No trouble for QMT powers}

In this section we consider again the criticisms raised for Megarian Actualism in Sect. 2. Taking each in turn I'll show that they each make an assumption about Megarian powers which does not apply to the quantitative cases just surveyed.

Bird's objection was that the Megarian is committed to trivialising modality (by having to deny B2). The objection is plausible if we take powers to be single-track (as Bird does), for then there is only one possible manifestation of any power-if it must manifest, then there can be no non-trivial possibilities about how instances of that power might otherwise behave. However, the objection fails once we acknowledge that powers may manifest in multiple different ways (corresponding to the values of a manifestation variable). An electric charge, for instance, may be manifesting some value of electrostatic force, but it remains possible for it to manifest another value and indeed it would do so were the electric field to change. The Megarian view just outlined does not trivialise modality, then, because it endorses the QMT view of powers, and no dispositionalist should suppose that a QMT view of powers renders modality trivial, since by necessity, there will always be some possible instances of manifestation which are not occurring.

Molnar's objection was that since Megarian powers can only be instantiated if their manifestations are instantiated as well, then they can't explain those manifestations. The objection is plausible so long as the entailment of instantiation is interpreted (as Molnar does) as one of asymmetrical ontological dependence. However, the interpretation is unreasonable. To begin with, there is no reason to suppose that power variables are ontologically dependent on their manifestation variables in this sense. After all, it is entirely consistent with the entailment of instantiation that the ontological dependence goes the other way around. This would be the case if, for example, one took the manifestation variable to be fully grounded in, the power variable (cf. Fine 2012).

But however we settle the relations of ontological dependence, no dispositionalist should suppose that just because, say, a determinate power of conductivity can't be instantiated by some object without it also exhibiting some value for current density, then the conductivity won't explain the current density. To see why, it's helpful to draw on a notion of 'dependency' other than that of existential entailment, and used widely in scientific contexts. This is the notion of causal or nomological dependence among variables in which the dependent variables are effects of the independent variables. All the variables in the characteristic equations given in Sect. 3 are clearly distinguishable into 'independent' and 'dependent' variables, with the characterised power variable falling among the former and the manifestation variable among the latter. We know, for example, that conductivity causally explains current density since, as with many power variables, it can be intervened on to change the manifestation variable, not to mention our evidence due to Markovian statistical correlations. 
It is this causal-nomic sense of dependence which scientists typically care about when, for example, they do regressions or causal analysis. Moreover, the causalnomic dependencies between power and manifestation variables constitute precisely the kind of explanatory relationships which Molnar protested cannot exist if Megarian Actualism is true. For example, a higher value of charge will be liable to manifest a higher force, all else being equal, for the simple reason that force and charge are proportional and charge is the independent variable. But of course, that is consistent with the existential kind of dependence of any determinate charge having to manifest some force, as the Megarian believes it must.

Moving on, Aristotle's objection was that conceiving of powers as Megarian makes their acquisition and loss conditions trivial and counterintuitive. This objection is plausible so long as one assumes that the Megarian must limit the instances where the power is instantiated. However, Megarian actualism was suggested in the case of QMT powers because it was seen to be plausible that manifestations are in fact more widely instanced. The acquisition and loss conditions for conductivity, charge, carrying capacity, etc. therefore remain precisely as any dispositionalist would anyway expect: conductivity is a result of the atomic structure and heat-energy of the material, charge is a state-independent intrinsic feature of elementary particles, carrying capacity is a result of the environment's size and nutritional content, and so on. Acquisition and loss conditions remain, under the Megarian view of QMT powers proposed, as informative as ever.

Nevertheless, one might be concerned that manifestations just do not stretch to precisely those cases we should wish to say the respective power is instanced. One would think this especially if one took zero-values of the manifestation variable to be instances where the power is not manifesting. As was mentioned at the end of the previous section, this concern effectively amounts to the the final issue raised in Sect. 2 that Megarian powers get the data wrong: power variables can exhibit zero-values of their manifestation variables, but zero-values just are not cases of manifestation. In response, the Megarian has two options. One (problematic) option is to limit the instances where a power is instantiated after all, specifically to those cases where manifestation does not take a zero value. But this seems to give rise to precisely Molnar's and Aristotle's original concerns that powers would not be able to explain their manifestations, because they would be dependent on them and would also thereby have counter-intuitive acquisition and loss conditions. The second and more plausible option is to argue that zero-values are in fact genuine instances of manifestation. In what remains of this section, I want to show why this is a reasonable thing to think.

Do we have any reason in the scientific cases to make an exception of the zerovalues, that is, to treat zero-values as indicating a genuine absence of manifestation? I doubt it. Insofar as powers enter into relationships described by the above kinds of characteristic equations, they unify and explain a set of modally robust behaviours which include and are indifferent to any putative uniqueness of manifestations of zero-values. Consequently, the interpretations given by both Kistler and Vetter of QMT powers make reference to the characteristic equation in defining the manifestation rather than any subset of values (e.g. those not including zero). Such further discrimination would seem, at the very least, grossly inelegant, if not arbitrary. 
The explanatory unity across all instances of the manifestation variable (including zero-values) is confirmed by the previously mentioned causal-nomic asymmetries signified by profiles of manipulation via intervention and statistical correlation. Causal modelling in scientific contexts works predominantly on the understanding that causal relations are foremostly relations among variables, and that relations of token causation between their values is derivative of those relations. A causal model consists of a set of variables and dependency relationships which give rise to statistical correlations and strategies for manipulation via intervention. From the perspective of constructing a model, however, there is no demarcation of zero-values, since the dependencies will typically remain regardless of where the zero-value is placed on a variable's scale. Things get more complicated when we introduce structural equations, which aim to relate the precise numerical values of the variables, but such equations can often be numerically adjusted to accommodate for any arbitrary change in the placement of the zero-value along a variable's scale. ${ }^{10}$

None of this should come as any surprise. Where we place the zero on our scales of measurement is often independent of the underlying properties being measured (e.g. for Celsius and Fahrenheit) and also often dependent on an arbitrary choice of reference frame (e.g. for velocity and momentum). Even when there are more principled reasons for placement, zero-values are not necessarily indicative of the lowest end of a scale. For instance, many magnitudes and vector quantities can take minus values (e.g. charge, force, acceleration, growth rate, current). These points alone can make it seem unjustified to treat a zero-value as a genuine absence of manifestation. More vividly, such treatment is also, on occasion, in tension with counting legitimate non-zero values as manifestations, since some scientifically relevant quantities are reciprocals of others (e.g. conductivity and resistivity); a zero-value for one is an infinity for the other so if we deny the reality of zero-value cases we'd also have to deny the realities of infinite values. Zero-values of manifestation also cannot be taken as an indication that the respective power is 'not doing anything'. For example, carrying capacity and population can take non-trivial positive finite values, but if they precisely match then the manifesting change in population will be zero. Similarly, the fact the net force experienced by a particle is zero might well be explained not by the lack of powers acting but by a balance of powers (e.g. of mass and charge, as in Millikan's oil-drop experiment). Points like these serve to show that, in some cases at least, denying the zero-values of a manifestation variable the status of a genuine instance of manifestation is without motivation.

Of course, zero-values of manifestation mean that a power might be less easy to discover. For example, a positively charged particle in the absence of an electrical field will not manifest any positive electrostatic force and so will be indistinguishable from a particle which is neutrally charged but otherwise identical. But we don't take manifestations of barely-detectable forces to be less real than easily detectable forces (1N isn't less real than $10 \mathrm{kN}$ ) and it's far from obvious that we should infer just from the continuum of increasing difficulty in detection that there is an ontological change

\footnotetext{
10 Some equations only hold over limited ranges of values (e.g. Hooke's law) but in none of the considered cases does this exclude the zero-value case.
} 
(from manifestation to no manifestation) just as the possibility of detection reaches precisely none.

The legitimacy of treating the zero-value of manifestation variable as genuine manifestation anyway enjoys more general defensibility. Notice that quantitative Powers are invoked not only to explain what something can do but also what it can't. For example, we explain why an electron does manifest a certain force by reference to its electric charge. But reference to that power also explains why it doesn't manifest another value of force: given the determinate power, and the fact the stimulus field is as it is, the force had to be as it was. Notice that exactly this kind of explanation is no less available in the zero-value case: we can explain why an electron cannot manifest anything other than a zero force by reference to the fact that it has electric charge, and not some other kind of electrostatic power. ${ }^{11}$ So, while explaining why a power manifests a zero-value can seem like an explanation of why it's not doing something, given that any other manifestation can be thought of in the same way, the case against zero-value manifestations subsides.

In fact, the case for real zero-values can and has already been made independently of dispositionalist concerns. Balashov (1999) provided a sequence of schematic arguments which show, when applied to certain quantities, that zero-value attributions are most naturally conceived as denoting a real property as opposed to an 'absence', 'privation' or 'hole in being'. For example, he pointed out that quarks and anti-quarks with non-zero spin can combine to form both zero and non-zero-valued mesons. Since all mesons obey the same Bosonic spin statistics it is natural to attribute spin-zero as a genuine property. By parity of reasoning, it is natural also to consider the zero-spin of the Higgs boson, which is not a composite, to have the same reality. If spin-zero bosons did not have integer spin (albeit zero-valued) Balashov points out that there would be no way to explain what unifies bosons. Moreover, the voluminous theoretical work on supersymmetry operates under the idea that bosons and fermions are united by a common symmetry in spin statistics, of which it is the integer/half-integer distinction which is crucial and not whether integer spins are zero-valued or not. Balashov applies similar reasoning to other quantities including charge, quark color and flavor, and (to a lesser degree) mass. ${ }^{12}$

In sum, there is a strong case for the legitimacy of treating zero-values of manifestation variables as genuine manifestations. One might worry that endorsing them will trivialise the attribution of powers, but this is not the case. If zero-valued manifestations are just as real as other values then there is an ontological difference between the absence of a manifestation of (e.g.) electrostatic force, which is attributable to abstract objects, and a zero-value of electrostatic force, like that experienced by a pos-

\footnotetext{
11 This is an instance of a more general kind of explanation given for why things happen in a certain way. For instance, we can explain why some organism failed to reproduce by reference to their sexual fecundity and lack of suitable mate. By referring to their power of sexual reproduction we imply the organism does not asexually reproduce and hence why it had no offspring given the poverty of an appropriate stimulus (thanks to Margarida Hermida for suggesting this example.)

12 Dispositionalists may note that Balashov takes his arguments for real zero-values to lend support to their position over categoricalism, since it's hard to see what could be the 'metaphysical stuff' of which a zero-valued categorical property could amount too. If the inference is justified, it would provide a further (admittedly methodologically spurious) argument for dispositionalists accepting zero-values, since it would provide them with further ammunition against their foes.
} 
itive charge in a zero-valued electrical field. Only some things are capable of exhibiting zero-values of electrostatic force, and being a concrete part of physical reality seems like a basic criterion. Whether this criterion is also sufficient depends, at least, on whether we want to say that (e.g.) neutrally charged particles also manifest a zerovalue of force as opposed to lacking any property of force. Here the Megarian has a choice: either say that neutrally charged particles do not have any power of charge, and so do not manifest electrostatic force of any value, or say that neutrally charged particles have a real zero-valued power of charge, and so manifest electrostatic force which is necessarily zero-valued. My inclination is to opt for the latter, perhaps even going so far as to attribute to all physical reality some value for every physical power (photons have zero-valued mass, protons zero-valued colour, etc.). After all, this seems like a natural generalisation of the kind of reasoning emerging here about zero-values in science. Charge (like force) is a quantity which takes values on a quantitative scale, and zero-values of charge (or any other power variable) seem to have just the same explanatory power as positive or negative values. Nevertheless, nothing I'll say here requires this. All the Megarian requires is that if there is a real power then there is a real manifestation. This strictly requires only the endorsement of real zero-valued manifestations, which I take to be reasonably justified.

The accommodation of zero-values among cases of manifestation of quantitative powers marks a response to the final complaint against Megarian Actualism mentioned in Sect. 2. This was the concern that Megarians have to oppose common-sense data that suggest that powers aren't always manifesting, for instance when they're not stimulated or when they're masked. In the case of QMT powers we saw that there are good reasons to think that there are no cases where the power is not manifesting since they will always be manifesting some value of the manifestation variable (Sect. 3). In particular, there seem to be good reasons to take the zero-value manifestations to be just as real as other values. The fact that a stimulus variable takes a zero value or that the power is masked in some way gives us no reason to deny any of this, since they won't prevent the fact that some value of the manifestation variable will be instanced. In the case of QMT powers, at least, the Megarian position is perfectly consistent with the data.

\section{Megarian Variable Actualism}

So far we've seen reason to think the quantitative powers represented in certain scientifically valuable characterising equations are appropriately characterised as Megarian. Nothing has been said to indicate that we should generalise to all powers; that is, to endorse the following thesis.

Megarian Variable Actualism. All powers must always be multiply directed at the values of (exhaustive) manifestation variables.

An endorsement of Megarian Variable Actualism (henceforth MVA) implies that all powers are QMT. But it is also an endorsement of Megarian Actualism, since if a power is directed at every value of an exhaustive variable, so that the power is manifesting 
as long as it instantiates one of them, it will be always manifesting. I have shown that MVA plausibly holds in the restricted domain of quantitative powers characterised by scientific equations. But can we generalise? I want here to suggest that there is a fairly straightforward way to extend the treatment of quantitative powers to all cases. I will also suggest that we have considerable reasons for doing so.

The path to generalisation is to observe first that paradigmatic manifestations, such as breaking for fragility, changing shape for malleability, making a sound for sonority, etc. are only single determinate values of more general exhaustive manifestation variables all of whose values the respective powers are directed towards. Let's focus on fragility. According to the proposal fragility is directed towards a manifestation variable $B$, which we might call 'degree-of-breaking'. $B$ takes mutually exclusive values which exhaust the range of possible states an object can be in with respect to the degree to which it is breaking. As with many manifestation variables, $B$ may be represented at different 'grains'. In the simplest case, $B$ is a binary variable where $B=0$ if the object exemplifying it is breaking to a null degree (and so remaining intact) and $B=1$ if the object is breaking to some positive degree. However we might also treat $B$ as a continuous variable which takes any value on the real number line between 0 and 1 (or some other arbitrary non-zero number), such that $B=0$ for some object if it is breaking to a null degree, $B=1$ if its being separated into its smallest component pieces (plasmarised) and $B$ takes a number in between with respect to how close it is to either of these two extremes (e.g. splitting, fracturing, shattering, atomising). ${ }^{13}$

We have then a general schema for accommodating any power within MVA. Step 1: where some arbitrary power $P$ (e.g. malleability, sonority) looks like it should be able to exist without manifesting some manifestation $M$ (e.g. changing shape, making a sound) locate a corresponding manifestation variable degree-of-M which takes $M$ as one of its values as well as other values which together exhaust the range of possible states corresponding to the degree to which it is $M$-ing. Specifically, degree-of $-M$ is to include the zero-value case in which we might naturally want to say that the power is 'not $M$-ing', although in this new idiom of degrees we'd do better to say the power is ' $M$-ing to the null degree'. Step 2: re-identify $P$ as a QMT power with all the values of degree-of- $M$ as its possible manifestations (malleability is directed towards all the possible values of degree-of-change-of-shape, sonority towards all the possible values of degree-of-making-a-sound, and so on). Since the degree-of- $M$ variable exhausts the possible states an object can be in with respect to the degree to which it is $M$-ing then instances of $P$ will always be manifesting.

Following the schema, we end up with a view of powers in general as QMT powers, directed to all the possible values of an exhaustive manifestation variable. The schema shows how it can be possible for the Megarian to admit the data that, e.g., fragile vases aren't always breaking, malleable clay is not always changing shape and sonorous metals aren't always making a sound (this was the concern at the end of Sect. 2). For according to MVA a fragile (malleable, sonorous) object is manifesting even when it is not doing these things. For instance, an object will be manifesting its fragility when not being struck or when being struck but housed in styrofoam because it will

\footnotetext{
13 These variables are quantities in that their determinate instances are ordered and have distance relations between each other (Eddon 2013).
} 
be exhibiting some degree of breaking (most likely, the null degree). That's all it takes to manifest under the proposed view.

I take it that the schema is straightforward enough. But given its evident departure from natural parlance, is there any value to thinking about everyday powers this way? Indeed there are. First, there are significant benefits from the greater continuity with scientific examples. This becomes especially clear when we represent the power itself as the value of a power variable for degree-of - $P$ along with the manifestation variable degree-of- $M$. For instance, fragility's directedness towards the values of $B$ can then be captured by a characterising equation with its own 'fragility function' $f$,

$$
B=f(F, \sigma)
$$

where $F$ is the variable degree-of-fragility, taking different values depending on how fragile something is, and $\sigma$ is the stimulus variable, taking different values of determinate stress. In typical contexts something's being fragile will correspond with instances of this function which correspond with particularly high values of $F$. Of course, the fragility function is likely to be vague, with no precise threshold above which instances of $F$ count as 'fragile'. But we do know quite a bit about what the function must be like. For instance, we know that $f$ must make both $F$ and $\sigma$ positively correlated with $B$. If $B$ is binary, we may assume that the function involves some step or sharp change of values of stress for which varying values of fragility will lead to positive degree of breaking. Comparatively, if $B$ takes more continuous values then there may be multiple steps or even a continuum for different phases of breaking.

Admittedly, the proposed characteristic equation for fragility (and other values of $F$ ) may not represent a law or principle of science, but it is lawlike in that it supports counterfactual reasoning and inductive inference. In particular, I see no reason to think that such an equation does not represent just the same unity and modal explanation of relationships among different determinate values of its variables as was argued by Kistler and Vetter are represented by the characteristic equations for quantitative powers (see Sect. 3). And I take it that similar remarks would go for other powers, such as malleability and sonority, each admitting of their own (potentially imprecise) power functions. ${ }^{14}$

The thought that powers' directedness in general is encoded in the logical structure of a function is not a new idea. Chakravartty remarks that it arguably contributes little more to the illumination of the conception of a power to say that 'we should think of a dispositional property [power] as the ontological correlate of a function, which takes an entity or collection of entities from one state of affairs to another' (2019, p. 14). To endorse MVA is effectively to make it possible to take this as literally as possible,

\footnotetext{
$\overline{14}$ Powers need not, in general, be represented this way, however. The power of a light-switch to illuminate a room (if we may talk of such a power at all, see below) may be highly contingent (e.g. on the circuit and suitable triggers) but does not clearly come in degrees as such. As mentioned in Sect. 4, MVA strictly only implies that manifestations must be instances of a variable. Obviously, if all properties (including manifestations) are powers, as pandispositionalists maintain, then we would have to reassess such powers: either there really is a degree to which something has a power to illuminate a room (which may be only a binary scale) or there is no such power at all.
} 
and there is significant value to representing powers this way. First, it allows us to be precise about what the nature of a power's influence on its manifestation variable is: for instance whether it follows a linear, smooth or sinusoidal curve, whether the correlations are positive, negative, chart a trough or a peak, etc. For many everyday powers, we may only have the slightest idea about how to answer these sorts of questions, but the fact we can ask them is indicative of the coherence and effectiveness of this form of representation. Second, treating powers like fragility as a determinate value (or a range of values) of a power variable allows us to move away from what is often an over-idealised discrete representation of the nature of powerful properties as something which objects either have or don't have (cf Vetter 2015). Third, it also allows us to consider 'opposing' powers, such as fragility and toughness, in a parsimonious and theoretically fruitful way by capturing them with the same function(s), e.g. a low degree of fragility $F$ may simply be identified as a high degree of toughness (cf. resistivity and conductivity). In sum, while it can seem at first glance excessive to represent everyday powers like fragility, malleability and sonority in the manner of a QMT power, thereby giving them greater continuity with the scientific examples, the representational benefits are significant. Anyone who would eschew the legitimacy of this manoeuvre will have to explain why the connections it illuminates among powers and the questions it raises about the manner of their directedness are so coherent.

So much for the benefits of continuity with science. There is also a more metaphysical benefit to endorsing MVA as a general treatment of powers. In perhaps the only contemporary defence of a form of dispositionalism consistent with Megarian Actualism (though this is not mentioned by the authors) Esfeld and Sasche (2011) argue that unless powers manifest 'spontaneously', by which they mean always and independently of a stimulus, then their nature can be hidden in some worlds.

There could then be a possible world in which the triggering [i.e. stimulus] conditions in question are absent. Consequently there could [...] be two types of properties $P$ and $P^{*}$ without the difference between them leading to different effects anywhere in the world. In other words, there could again be a difference that does not make a difference. (pp. 37-38)

This consequence is something of an embarrassment for dispositionalists who often motivate a preference for a powers-ontology over the rival categoricalist ontology by reference to the distinguishability of properties. According to a categoricalist ontology properties have no non-trivial modal connections. They are, therefore, individuated by 'quiddities' (a 'such-ness' for properties equivalent to haecceities' 'this-ness' for particulars). In motivating their alternative powers ontology, Kistler (2002), Mumford (2004), Bird (2007), Esfeld and Sasche (2011) have pointed out that if only quiddities individuate two properties $F$ and $G$ then there can be two qualitatively identical worlds $w_{1}$ and $w_{2}$ distinguished only by the fact that the causal role $F$ and $G$ have in $w_{1}$ is swapped over in $w_{2}$. Moreover, the quiddistically individuated properties seem compatible with a world $w_{3}$ in which $F$ and $G$ have an identical causal role. These results mean that there is a distinction in reality which can have no testable difference. For instance, there is nothing we could do to determine that we are in $w_{1}$ as opposed to $w_{2}$ or $w_{3}$ (see Schaffer 2005, for a discussion about how concerning this really is). The 
issue identified by Esfeld and Sasche, however, is that the possibility of unmanifested powers gives rise to precisely the same concern. For if powers can go unmanifested, then its possible that there is no evidence of their instantiation.

The only way of avoiding this issue, Esfeld and Sasche suggest, is to admit that powers always manifest. ${ }^{15}$ This is a feature of fundamental physical properties which they argue is anyway plausible. For example, they point out that charge generates an electromagnetic field spontaneously, irrespective of the presence of other objects. Hence there is no danger of charge being possibly indistinguishable from, say, mass (which generates a different field). An endorsement of MVA therefore benefits from making this claim for all powers.

Of course, from the standpoint of more orthodox interpretations of powers MVA brings with it some re-conceptualisation. I think enough has been said here to make that seem relatively easy in the case of the quantitative powers referenced across the sciences and at the very least highly defensible in the case of everyday conventional powers like fragility, malleability and sonority. Things are more tricky when it comes to highly contextual power-ascriptions, as we might give to a push-switch for turning a light on. ${ }^{16}$ If we are to capture this under MVA we would have to posit a binary variable property for the state of the light; the switch would then be ascribed a power multiply directed at the values of that variable. But it can seem very odd to posit such a power. Push-switches, we might think, should be thought of as doing something only when 'on', i.e. closing a circuit and causing (e.g.) a light to illuminate; in their 'off' state push-switches aren't doing anything at all.

In response the defender of MVA might say one of two things. One option is to deny the existence of the power of the push-switch to turn a light on, or else suggest the property is a mere 'disposition' (Bird 2016). There are a number of reasons one could give for this, perhaps the disposition is too contextually defined, not scientifically generalisable enough or not natural enough. The other option is to double-down. The push-switch genuinely has such a multiply-directed power, as would be represented more saliently in a causal graph of the scenario according to which interventions can be performed on the push-switch to manipulate the variable state of the light ( $a$ la, Woodward 2003). Were we more inclined to read our ontology of causal relations directly from such diagrams, where causal relationships hold between variables rather than determinate events, then this might seem perfectly legitimate (cf Sect. 4). Either way, the Megarian need not expect one kind of response to work in all cases. It might be that a mixture of trimming down our ontological commitment to powers and adopting a more embracing interpretation of type-level causation gets the most plausible results.

In sum, MVA has plenty to support it. It promises both to make everyday powers continuous with those posited in scientific contexts and to avoid the possibility of hidden powers. Moreover, MVA has a number of ways to assuage concern over particular examples which don't seem to fit so easily within the framework. Ultimately, however, whether one endorses MVA or not, the case for Megarian QMT powers in the scientific context remains.

\footnotetext{
15 Contrary to their claim, this needn't be because the manifestation is independent of stimuli. For instance, it may be as a consequence of the stimuli being necessarily present.

16 Thanks to an anonymous reviewer for suggesting this example and an objection to it on the basis of unnaturalness.
} 


\section{Conclusion}

Almost all accounts of dispositionalism to date have assumed the central dispositionalist platitude that powers need not manifest. Moreover, its endorsement has been accompanied by some weighty objections to the contrary position of Megarian Actualism. However, by focusing attention on the quantitative powers in scientific equations a strong case can be made that at least some powers always manifest. This is for the simple reason that such powers are only plausibly understood as being directed at every value (including the zero-values) of their respective exhaustive manifestation variables. Moreover, understanding quantitative powers in this way reveals the failure of the objections to Megarian Actualism to apply in general. I showed how we can extend the treatment of quantitative powers in scientific contexts to more 'everyday' powers by treating the latter as being characterised by equations in a similar way. Such an extension amounts to a general and scientifically informed view of powers, Megarian Variable Actualism, which has significant benefits worthy of attention among dispositionalists. Pace Aristotle, endorsing Megarian Actualism might therefore not be such buffoonery after all. ${ }^{17}$

Acknowledgements I would like to thank the editors for inviting me to submit, to the department at Bristol University for their questions to my presentation of these ideas and to Sam Kimpton-Nye, Andrea Raimondi, Francesca Bellazzi and two anonymous reviewers for their helpful comments on various drafts. This work was carried out as part of the MetaScience project funded by the European Research Council (ERC) under the European Union's Horizon 2020 research and innovation programme (Grant Agreement No. 771509).

Open Access This article is licensed under a Creative Commons Attribution 4.0 International License, which permits use, sharing, adaptation, distribution and reproduction in any medium or format, as long as you give appropriate credit to the original author(s) and the source, provide a link to the Creative Commons licence, and indicate if changes were made. The images or other third party material in this article are included in the article's Creative Commons licence, unless indicated otherwise in a credit line to the material. If material is not included in the article's Creative Commons licence and your intended use is not permitted by statutory regulation or exceeds the permitted use, you will need to obtain permission directly from the copyright holder. To view a copy of this licence, visit http://creativecommons.org/licenses/by/4.0/.

\section{References}

Aristotle. (1994). Categories. E. M. Edghill (trans.). http://classics.mit.edu/Aristotle/categories.1.1.html. Aristotle. (1998). Metaphysics. Lawson-Tancred, H. (trans.). Penguin.

Balashov, Y. (1999). Zero-value physical quantities. Synthese, 119(3), 253-286.

Bird, A. (2007). Nature's Metaphysics: Laws and Properties. Oxford: Oxford Clarendon Press.

Bird, A. (2016). Overpowering: How the powers ontology has overreached itself. Mind, 125(498), 341-383. Cartwright, N. (1983). How the laws of physics lie. Oxford: Oxford University Press.

Cartwright, N. (1999). The dappled world: A study of the boundaries of science. Cambridge: Cambridge University Press.

Cartwright, N. (2019). Nature, the artful modeler. Chicago: Open Court Publishing.

\footnotetext{
17 I would like to thank the editors for inviting me to submit, to the department at Bristol University for their questions to my presentation of these ideas and to Sam Kimpton-Nye, Andrea Raimondi, Francesca Bellazzi and two anonymous reviewers for their helpful comments on various drafts. This work was carried out as part of the MetaScience project funded by the European Research Council (ERC) under the European Union's Horizon 2020 research and innovation programme (grant agreement No. 771509).
} 
Chakravartty, A. (2003). The dispositional essentialist view of properties and laws. International Journal of Philosophical Studies, 11(4), 393-413.

Chakravartty, A. (2019). Physics, metaphysics, dispositions, and symmetries-Á la French. Studies in History and Philosophy of Science Part A, 74, 10-15.

Eddon, M. (2013). Quantitative properties. Philosophy Compass, 8(7), 633-645.

Ellis, B. (2000). Causal laws and singular causation. Philosophy and Phenomenological Research, 16(2), $329-351$.

Ellis, B., \& Lierse, C. (1994). Dispositional essentialism. Australian Journal of Philosophy, 72, 27-45.

Esfeld, M., \& Sasche, C. (2011). Conservative reductionism. London: Routledge.

Fine, K. (2012). Guide to ground. In F. Correia \& B. Schneider (Eds.), Metaphysical grounding: Understanding the structure of reality (pp. 37-80). Cambridge: Cambridge University Press.

Handfield, T. (2010). Dispositions, manifestations, and causal structure. In A. Marmodoro (Ed.), The metaphysics of powers: Their grounding and their manifestations. London: Routledge.

Harré, R., \& Madden, E. (1974). Causal powers. Oxford: Oxford University Press.

Heil, J. (2003). From an ontological point of view. Oxford: Oxford Clarendon Press.

Heil, J. (2005). Dispositions. Synthese, 144(3), 343-356.

Hüttemann, A. (2004). What's wrong with microphysicalism?. London: Routledge.

Hüttemann, A. (2014). Ceteris paribus laws in physics. Erkenntnis, 79, 1715-1728.

Jenkins, C. S., \& Nolan, D. (2012). Disposition impossible. Noûs, 46(4), 732-753.

Johnston, M. (1992). How to speak of the colors. Philosophical Studies, 68, 221-263.

Kistler, M. (2002). The causal criterion of reality and the necessity of laws of nature. Metaphysica, 3, 57-86.

Kistler, M. (2012). Chapter 8: Powerful properties and the causal basis for dispositions. In A. Bird, B. Ellis, \& H. Sankey (Eds.), Properties, powers and structures (pp. 119-138). London: Routledge.

Kistler, M. (2020). Chapter 11: Powers, dispositions and laws of nature. In A. Meincke (Ed.), Dispositionalism: Perspectives from the metaphysics and the philosophy of science (pp. 171-188). Berlin: Springer.

Lowe, E. J. (2010). On the individuation of powers. In A. Marmodoro (Ed.), Metaphysics of powers-their grounding and their manifestations (pp. 8-26). London: Routledge.

Martin, C. B. (1994). Dispositions and Conditionals. The Philosophical Quarterly, 44, 1-8.

Martin, C. B. (2008). The Mind in Nature. Oxford: Oxford Clarendon Press.

Martin, C. B., \& Pfeifer, K. (1986). Intentionality and the non-psychological. Philosophy and Phenomenological Research, 46(4), 531-554.

McKitrick, J. (2010). Chapter 5: Manifestations as effects. In A. Marmodoro (Ed.), The metaphysics of powers: Their grounding and their manifestations (pp. 73-83). London: Routledge.

Molnar, G. (2003). Powers: A study in metaphysics. Oxford: Oxford University Press.

Mumford, S. (2004). Laws in nature. London: Routledge.

Place, U. (1999). Intentionality and the physical: A reply to Mumford. Philosophical Quarterly, 49(195), 225-231.

Schaffer, J. (2005). Quidditistic knowledge. Philosophical Studies, 123, 1-32.

Shoemaker, S. (1979). Identity, properties, and causality. Midwest Studies in the Philosophy, 4(1), 321-342.

Swoyer, C. (1982). The nature of laws of nature. Australasian Journal of Philosophy, 60(3), 203-223.

Tugby, M. (2013). Platonic dispositionalism. Mind, 122(486), 451-480.

Vetter, B. (2012). Chapter 12: Dispositional essentialism and the laws of nature. In A. Bird, B. Ellis, \& H. Sankey (Eds.), Properties, powers and structures (pp. 201-215). London: Routledge.

Vetter, B. (2015). Potentiality: From dispositions to modality. Oxford: Oxford University Press.

Williams, N. (2011). Putting powers back on multi-track. Philosophia, 39, 581-595.

Woodward, J. (2003). Making things happen. Oxford: Oxford University Press.

Publisher's Note Springer Nature remains neutral with regard to jurisdictional claims in published maps and institutional affiliations. 